\title{
Evaluación educativa utilizando rúbrica: un desafio para docentes y estudiantes universitarios
}

Manuela Raposo-Rivas

Universidad de Vigo, España

mraposo@uvigo.es
$M^{a}$ Esther Martínez-Figueira

Universidad de Vigo, España esthermf@uvigo.es

\section{Resumen}

Presentamos una nueva propuesta metodológica en la práctica evaluadora que está tomando impulso en las aulas universitarias españolas: la integración de la rúbrica como herramienta de apoyo para la evaluación, tanto por parte del docente como de los estudiantes, a la hora de realizar evaluación de pares y autoevaluación. Los datos fueron obtenidos durante el curso académico 2012-13, en la materia Nuevas Tecnologías aplicadas a la Educación Infantil, perteneciente al primer curso de la formación inicial de futuros docentes de Educación Infantil (3-6 años). Se trabajó con 71 estudiantes distribuidos en 7 grupos experimentales (que utilizan la rúbrica) y 6 de control (que no la utilizan) en diferentes situaciones de aprendizaje. Se pretendió constatar su influencia tanto en el dominio de contenidos como en el desarrollo de competencias e implicar al alumnado en el proceso de evaluación. Los resultados manifiestan que estamos ante un instrumento que contribuye a la educación infantil y que los estudiantes están satisfechos con su uso.

\section{Palabras clave}

Pedagogía universitaria, evaluación del aprendizaje, métodos de evaluación, formación de profesores, evaluación educativa (Fuente: Tesauro de la Unesco).

Recepción: 2014-01-31 | Envío a pares: 2014-06-19 | Aceptación por pares: 2014-10-27 | Aprobación: 2014-11-09 DOI: 10.5294/edu.2014.17.3.6

Para citar este artículo / To reference this article / Para citar este artigo

Raposo-Rivas, M. y Matínez-Figueira, M. E. (2014). Evaluación educativa utilizando rúbrica: un desafío para docentes y estudiantes universitarios. Educ. Educ. Vol. 17, No. 3, 499-513. DOI: 10.5294/edu.2014.17.3.6 


\title{
Using Rubrics to Evaluate Education: A Challenge for University Teachers and Students
}

\begin{abstract}
Rubrics are a new grading method that is gaining acceptance in Spanish university classrooms as an assessment-support tool for both teachers and students in peer review and self-evaluation processes. The data in this study were obtained during the 2012-13 academic year, from the course on New Technologies for Pre-school Education, which is the first subject taught in initial training for future teachers at the preschool level (children ages 3-6). The sample included 71 students. They were divided into seven experimental groups (that used a rubric) and six control groups (that did not) in different learning situations. The objective was to verify the effect of rubrics on mastering contents and on developing skills and involving students in the evaluation process. The results show this tool contributes to pre-school education and students are satisfied with its use.
\end{abstract}

Keywords

College teaching, learning assessment, evaluation methods, teacher training, evaluating education (Source: Unesco Thesaurus). 


\section{Avaliação educativa que utiliza rubrica: um desafio para docentes e estudantes universitários}

\section{Resumo}

Apresentamos uma nova proposta metodológica na prática avaliadora que está ganhando impulso nas salas de aula universitárias espanholas: a integração da rubrica como ferramenta de apoio para a avaliação, tanto por parte do docente quanto dos estudantes, na hora de realizar avaliação de pares e autoavaliação. Os dados foram obtidos durante o curso acadêmico 2012-2013, na matéria Novas Tecnologias Aplicadas à Educação Infantil, pertencente ao primeiro curso da formação inicial de futuros docentes de Educação Infantil (de 3 a 6 anos). Trabalhou-se com 71 com estudantes distribuidos em sete grupos experimentais (que utilizam a rubrica) e seis de controle (que não a utilizam) em diferentes situações de aprendizagem. Pretendeu-se constatar sua influência tanto no domínio de conteúdos quanto no desenvolvimento de competências e implicar o alunado no processo de avaliação. Os resultados demonstram que estamos diante de um instrumento que contribui para a educação infantil e que os estudantes estão satisfeitos com seu uso.

\section{Palavras chave}

Pedagogia universitária, avaliação da aprendizagem, métodos de avaliação, formação de professores, avaliação educativa (Fonte: Tesauro da Unesco). 


\section{Introducción: la evaluación de pares, la autoevaluación y la rúbrica en la universidad}

La convergencia de una parte importante de los sistemas universitarios europeos como respuesta a los principios del llamado "proceso de Bolonia", ha supuesto grandes cambios en la universidad española, no solo en la estructura de las titulaciones sino también en los procesos de enseñanza y aprendizaje que se desarrollan sobre dos pilares fundamentales: la formación basada en competencias junto con la autonomía y autorregulación del aprendizaje. Ambos aspectos tienen repercusiones directas sobre las prácticas evaluadoras que se han de llevar a cabo, lo que ha exigido la renovación de estrategias, herramientas e instrumentos de evaluación, lo que coincide con la opinión de Biggs (1996) sobre los procedimientos de evaluación, a los que considera como determinantes del aprendizaje de los estudiantes en mayor medida que los objetivos del currículo y los métodos de enseñanza.

Así, tal como indica Mora (2004), la universidad no es la misma de antes de la reforma. Se constituye como una nueva institución que debe adoptar nuevos objetivos y acoger mecanismos flexibles de adaptación continua a esos objetivos. El nuevo modelo educativo está centrado más en el estudiante y su aprendizaje, por tanto, según este autor, más en los resultados de ese aprendizaje, que en cómo se ha realizado el proceso.

Entre las implicaciones que un modelo de formación basado en competencias posee sobre los aspectos evaluativos, destacamos fundamentalmente aspectos relativos al objetivo, la finalidad y las estrategias de recogida de información.

- El objetivo de la evaluación de los estudiantes se orienta hacia la mejora del proceso de aprendizaje (evaluación formativa), y, en consecuencia, hacia un modelo de evaluación continua (Olmos-Migueláñez y RodríguezConde, 2010). La evaluación constituye un ele- mento integrado en el proceso de aprendizaje del estudiante, desde el inicio del proceso hasta el momento de obtener una calificación final (Delgado et al., 2006).

- Se desarrolla la capacidad de autorregulación (Carneiro et al., 2011) del propio proceso de aprendizaje, entendida como "la capacidad para autodirigir los factores cognitivos, motivacionales y afectivos que intervienen en el proceso de aprendizaje en pos de alcanzar una meta académica previamente fijada" (Daura, 2013, p. 122). Desde este punto de vista, las tareas del profesor llevarían a plantear la necesidad de articular mecanismos de feedback valiosos (Olmos-Migueláñez, 2008), que ayuden a los estudiantes en su proceso de aprendizaje, o lo que es lo mismo, a establecer mecanismos y estrategias que ayuden al alumnado a tomar conciencia de qué aprende y cómo lo hace.

- La evaluación basada en resultados de aprendizaje, requiere de una amplia variedad de estrategias de recogida de información que permitan analizar lo que el estudiante sabe, sabe hacer, cómo resuelve una situación y cuál es su actitud en cada caso.

Al mismo tiempo, parece fundamental la participación del estudiante en los procesos de evaluación, ya que, siguiendo a Bordas y Cabrera (2001), es una de las mejores garantías de utilidad para el aprendizaje y el aprendizaje de la evaluación: existe una responsabilidad compartida; se busca un consenso de diferentes intereses, valores y puntos de vista; se enfatiza la cooperación y colaboración en el transcurso del proceso de aprendizaje; el estudiante como evaluador es activo y cooperativo en su evaluación, al mismo tiempo que aprende a conocer y dirigir su proceso de aprendizaje.

En esta misma línea, están las premisas enunciadas por Weimer (2002) para ayudar a los profesores en el diseño de actividades de fomento de la 
autorregulación. Según el autor, una de las cuestiones fundamentales es el balance de poder, esto es, que los profesores otorgan un mayor protagonismo al alumno en su proceso de aprendizaje, así colaboran más activamente en las decisiones sobre aspectos como normas de funcionamiento, evaluación y metodología; este poder se distribuirá de acuerdo con la madurez, la habilidad y la capacidad de los alumnos para llevarlo a cabo. Otra tiene que ver con los procesos y las funciones de la evaluación, de tal forma que esta no se traduce solo en la medición de los niveles finales de aprendizaje y rendimiento del alumno, sino que se amplía al englobar todo el proceso de aprendizaje.

Así, la participación en los procesos de evaluación puede tomar forma, por ejemplo, en estrategias como la evaluación entre iguales y la autoevaluación, por poseer estas mayores oportunidades para el aprendizaje. Siguiendo a Ibarra, Rodríguez y Gómez (2012), la evaluación entre iguales puede entenderse como una forma específica de aprendizaje colaborativo en el que los aprendices realizan una valoración sobre el proceso o producto de aprendizaje de todos o de algún estudiante o grupo de estudiantes. Puede ser intragrupo, la cual está basada en la evaluación dentro de los grupos de trabajo donde cada participante o grupo valora el trabajo realizado por sus compañeros de forma individual o colectiva durante un proyecto común. Otra modalidad es la evaluación intergrupo, que sería la realizada entre grupos, también de forma individual o por equipos, con la que se valora el trabajo realizado por los distintos grupos. Y la evaluación individual, donde los estudiantes evalúan el proceso o producto del aprendizaje individual de sus iguales. Sea bajo una modalidad u otra, estos mismos autores destacan una serie de ventajas que los estudiantes encuentran al participar en tareas de evaluación entre iguales, como pueden ser: a) mejora de los procesos y productos del aprendizaje pues ayuda a estructurar dichos procesos, incrementando el aprendizaje y el rendimiento, y se convierte en un incentivo para mejorar el trabajo grupal y el propio esfuerzo, además de estimular el pensamiento y el aprendizaje profundo y crítico; b) desarrollo de estrategias interpersonales y habilidades sociales ya que los estudiantes perciben que desarrollan un mayor grado de empatía y competencias relacionadas con el trabajo en equipo y la cooperación; c) mejora en la capacidad de realizar juicios y evaluar pues los estudiantes que participan en experiencias de evaluación entre iguales van adquiriendo mayor confianza y destreza a la hora de realizar juicios y valoraciones; d) importancia para el desarrollo de la carrera profesional en competencias relacionadas con el trabajo en equipo, la empatía o la valoración del trabajo tanto propio como ajeno; e) desarrollo de competencias, entre ellas pensamiento reflexivo, crítico e independiente; diferentes soluciones a distintos problemas; aprendizaje autónomo; mayor capacidad de discusión y negociación; motiva el pensamiento, e incrementa el aprendizaje y la confianza.

Por su parte, Fallows y Chandramohan (2001) destacan tres tipos de dificultades a la hora de poner en práctica la evaluación entre iguales. La primera, de tipo conceptual, se refiere a que es necesario asegurar un cierto equilibrio entre la evaluación realizada por el tutor, que suele centrarse más en la calidad académica del trabajo realizado, y la de los estudiantes, que se centran y valoran el esfuerzo realizado para efectuar el trabajo. Un segundo de tipo institucional, pues las cuestiones que emanan de la institución, desde los recursos con los que cuenta, hasta las normas establecidas en dicha institución, pueden condicionar el proceso de evaluación. Por último, las de tipo relacional, que se explican porque la evaluación entre compañeros modifica las relaciones entre los participantes y diversifica la jerarquía tradicional de la evaluación educativa, promoviendo cambios en los roles y las responsabilidades de estudiantes y tutores, los cuales pueden cuestionar o dificultar la asunción de estas nuevas responsabilidades/roles.

Con independencia de sus ventajas e inconvenientes, Ibarra et al. (2012) confirman que la 
evaluación entre iguales es un buen procedimiento para contrastar los resultados de la autoevaluación y contribuir a que los juicios de los estudiantes sobre su propio aprendizaje sean cada vez más ajustados, ayudando así a desarrollar la capacidad de autorregulación y de aprendizaje autónomo a lo largo de la vida. Además, como indican Valenzuela y Pérez (2013), la autoevaluación, referida a la comparación de los resultados de rendimiento con una norma o meta, es una de las actividades más habituales que los docentes utilizan para fomentar en los estudiantes el uso de procesos autorreguladores.

Por otro lado, diferentes autores coinciden en reconocer las posibilidades de la rúbrica para la evaluación (Ahumada, 2005; Bartolomé, MartínezFigueira y Tellado-González, 2014, Blanco, 2008; Cebrián, 2008, 2009, 2012, 2014; Cebrián, Serrano y Ruiz, 2014; Etxabe, Aranguren y Losada, 2011; Hafner y Hafner, 2003; Martínez-Figueira, Tellado y Raposo-Rivas, 2013; Raposo-Rivas y Martínez-Figueira, 2011; Tierney y Simon, 2004; Wamba et al., 2007); estos autores insisten en que permite la orientación y evaluación en la práctica educativa.

Particularmente, es de destacar su valor en la autoevaluación de los aprendizajes por los estudiantes, ya que los guía, fomenta el aprendizaje cooperativo y constituye una herramienta ágil, útil y coherente que impulsa el aprendizaje. Asimismo, le permite a los docentes realizar un seguimiento y evaluación de las competencias adquiridas por los estudiantes de una manera más sistematizada mediante la utilización de indicadores que miden su progreso, creando una evaluación más objetiva y consistente a través de la clarificación de los criterios por valorar en términos específicos.

Por su parte, al alumnado le permite tener a su disposición las pautas explícitas de evaluación, siendo conscientes de los aspectos que serán objeto de valoración y del peso que tienen en la calificación global. Al respecto, Stevens y Levi (2005) van más allá y afirman que el empleo de rúbricas no solo favo- rece una evaluación más sistematizada por parte del docente, sino que estas son una herramienta de extraordinario valor para el desarrollo de competencias de monitorización, autoevaluación y evaluación entre pares, contribuyendo a un mayor entendimiento del propio proceso de aprendizaje y, en definitiva, a una mayor autonomía y autorregulación del proceso de aprendizaje del estudiante.

\section{El marco de investigación}

Como se indicó, el estudio realizado se enmarca en la investigación "Servicio federado de e-rúbrica para la evaluación de aprendizajes universitarios", con la que se desarrolló, exploró y evaluó el alcance educativo de las rúbricas electrónicas (e-rúbricas)' en diversos contextos de enseñanza universitaria, diferentes materias, tipo de presencialidad, modalidad de enseñanza y áreas de conocimiento dispares (Cebrián, 2011). Entre sus objetivos específicos figura el experimentar una metodología basada en la evaluación entre pares y la autoevaluación por los propios estudiantes a través de e-rúbricas, comparando estas con la del docente.

Se integra la rúbrica como herramienta de apoyo para la evaluación educativa independientemente de si quien la realiza es el docente, el estudiante a sí mismo (self-evaluation) o a otros compañeros (peer-evaluation). En el caso de nuestra universidad, una de las materias que participa en dicha investigación experimentando con el uso de este recurso es Nuevas Tecnologías aplicadas a la Educación Infantil, de la titulación de graduado en Educación Infantil. Aquí nos centramos en el seguimiento llevado a cabo en las actividades planteadas en las sesiones teóricas de esta materia, perteneciente al primer curso de la formación inicial de docentes de Educación Infantil (3-6 años). Analizamos el uso

1 Según se recoge en su página web (http://erubrica.org), se entiende la rúbrica como una herramienta y una metodología de evaluación formativa basada en competencias, que ayuda en la comunicación evaluativa entre docentes y estudiantes, al mismo tiempo que facilita la gestión de la autoevaluación de los aprendizajes por parte de estos últimos. 
de la rúbrica en la evaluación de competencias relacionadas con el dominio de los contenidos, con un enfoque que implica no solo al docente sino también a los estudiantes, participantes en procesos de reflexión y evaluación sobre su aprendizaje y el de sus iguales.
A continuación mostramos una ficha del estudio llevado a cabo durante el curso académico 20122013 en la citada materia, sintetizando el contexto, los agentes, los objetivos, la metodología, las estrategias de recogida de información, el procedimiento de análisis de datos y las conclusiones.

\section{Tabla 1. Sintesis del estudio realizado sobre evaluación educativa con rúbricas}

\begin{tabular}{|c|}
\hline Contexto \\
\hline $\begin{array}{l}\text { Nuevas Tecnologías aplicadas a la Educación Infantil, Facultad de Ciencias de la Educación y del Deporte, Universidad de } \\
\text { Vigo (España). }\end{array}$ \\
\hline Agentes implicados \\
\hline $\begin{array}{l}71 \text { estudiantes de la materia ( } 1^{\circ} \text { de Educación Infantil, curso académico 2012-2013), distribuidos en } 7 \text { grupos experimen- } \\
\text { tales ( } 38 \text { alumnos/as) y } 6 \text { grupos de control (33 alumnos/as). Cada individuo permanece en el mismo grupo a lo largo } \\
\text { de todo el cuatrimestre. } \\
\text { - } \quad \text { La docente de la materia. } \\
\text { - Los asesores especialistas en rúbrica: equipo de investigación del proyecto l+D+i. }\end{array}$ \\
\hline Objetivos de investigación específicos \\
\hline $\begin{array}{l}\text { - Experimentar nuevas formas de evaluación en la materia: evaluación docente, autoevaluación y evaluación de pares. } \\
\text { - Apreciar el uso de la rúbrica como herramienta de apoyo para la evaluación educativa. } \\
\text { - Constatar la influencia del instrumento en la evaluación, tanto en el dominio de contenidos como en el desarrollo de la } \\
\text { competencia "trabajo en equipo"y de "evaluación". }\end{array}$ \\
\hline Objetivos de formación \\
\hline $\begin{array}{l}\text { - Implicar al alumnado en el proceso de evaluación. } \\
\text { - Experimentar, como futuros docentes, nuevas formas de evaluación. } \\
\text { - Desarrollar la autonomía, responsabilidad, capacidad de reflexión y de autorregulación del aprendizaje. } \\
\text { - Aprender a trabajar en equipo. }\end{array}$ \\
\hline Metodología \\
\hline Se trata de un estudio cuasi experimental enmarcado dentro de una experiencia de investigación-acción. \\
\hline Estrategias de recogida de información \\
\hline $\begin{array}{l}\text { - Rúbricas de evaluación de contenidos para cada una de las actividades específicas de la materia. } \\
\text { - Rúbricas de autoevaluación de contenidos para cada una de las actividades específicas de la materia. } \\
\text { - Diario de la o del docente. } \\
\text { - Blog-portafolio del alumnado. } \\
\text { - Notas de campo del equipo asesor. } \\
\text { - Cuestionario final sobre la valoración de la experiencia. }\end{array}$ \\
\hline Procedimientos de análisis de datos \\
\hline $\begin{array}{l}\text { - Sobre la información de carácter cualitativo se han aplicado procedimientos de análisis interpretativo. } \\
\text { - Los análisis cuantitativos realizados sobre los resultados de las rúbricas y las respuestas de los estudiantes al cuestionario } \\
\text { han sido de carácter descriptivo (frecuencias, porcentajes y medidas de tendencia central) mediante el programa } \\
\text { informático SPSS para Windows. }\end{array}$ \\
\hline Conclusiones y utilidad de los hallazgos \\
\hline $\begin{array}{l}\text { En la elaboración de conclusiones y difusión de los hallazgos se han tenido en cuenta los objetivos de investigación y formación } \\
\text { propuestos, y se ha documentado la respuesta a cada uno de ellos a partir de la información recabada con los instrumentos. } \\
\text { Los hallazgos de este estudio han permitido recoger datos que facilitan la toma de decisiones para la mejora de experiencias } \\
\text { de este tipo y la recomendación de seguir utilizando este instrumento como estrategia para una evaluación formativa. }\end{array}$ \\
\hline
\end{tabular}

Fuente: adaptado de Martínez-Figueira, Tellado-González y Raposo-Rivas (2013). 
Coherente con el diseño de la investigación (Cebrián, 2011), este trabajo responde a un estudio de tipo cuasi experimental, en el que se distribuye a la totalidad del alumnado asistente a clase en grupos experimentales y grupos control, para poder investigar sobre las diferencias existentes en la evaluación. Así, el grupo experimental utiliza la rúbrica tanto para la evaluación de pares, aquella que realiza cada estudiante al trabajo o proyecto de un equipo, como para la autoevaluación, aquella que realiza de su propio trabajo; además, la evaluación que el profesorado lleva a cabo se basa en el mismo instrumento. Por otra parte, el grupo control realiza la evaluación de esas mismas tareas sin ningún instrumento guía. En este trabajo nos centramos en la comparación entre las evaluaciones llevadas a cabo en las cuatro actividades objeto de evaluación realizadas en las sesiones teóricas de la materia según los datos obtenidos en los grupos experimentales.

Dichas actividades compartían características semejantes para evitar variables distorsionantes a la hora de interpretar los datos. Son actividades monográficas de aula, con un nivel de dificultad y duración en el planteamiento, resolución y evaluación similares. Además, todas ellas deberían potenciar el dominio de habilidades y contenidos, que en este caso concreto fueron:

- Competencias del estudiante para una enseñanza innovadora con tecnología.

- Análisis didáctico y lectura crítica de un spot publicitario.

- El vídeo didáctico: ficha para su selección y clasificación.

- Valoración técnica y didáctica de un recurso multimedia: integración en enseñanzas obligatorias.

Para llevar a cabo el proceso de evaluación formativa y dirigida al aprendizaje, el procedimiento seguido se ha mantenido a lo largo del cuatrimes- tre: se realiza una actividad en cada mes del mismo y la docente la ejemplifica proponiendo otra similar que se realizará en equipos de cinco o seis personas en un tiempo determinado. Una vez terminada, cada equipo la entrega a través de la plataforma de teleformación de la Universidad. A continuación, cada equipo experimental cubre la rúbrica electrónica, realizando así la autoevaluación y evaluación de las respuestas de sus compañeros. Mientras, los grupos control realizan también un ejercicio de autoevaluación y evaluación de pares, pero respondiendo a preguntas generales que ayudan a orientar el proceso. Antes de terminar la sesión, la docente proporciona la solución de la actividad y deja un tiempo para la discusión.

\section{Resultados y discusión del estudio}

El nivel de rendimiento de cada actividad se evaluó con una escala de seis puntos en la que: No se muestra $=0$, Insuficiente $=1$, Mejorable $=2$, Aceptable $=3$, Considerable $=4$ y Excelente $=5$. La puntuación máxima para cada actividad fue de 2,5 puntos, dando una puntuación máxima total de 10. Esta puntuación representó el 10\% de la nota final de la asignatura. Si representamos gráficamente los resultados globales de las actividades vemos que los grupos experimentales se aproximan más a la máxima nota posible que los controles (gráfico 1). Se percibe, además, que tres de los grupos experimentales consiguen la nota máxima (10 puntos), mientras que ninguno de los controles la alcanza y que, incluso las puntuaciones mínimas, son más altas en los primeros que en los segundos.

Por limitaciones de espacio en este apartado se muestran los resultados solo de los grupos experimentales obtenidos en términos de rendimiento en la materia y de la evaluación del contenido utilizando la rúbrica en cada una de las cuatro actividades realizadas, tanto por parte del docente como en la autoevaluación y evaluación de pares realizada por los estudiantes (gráficos 2 a 5). 
Gráfico 1. Puntuación obtenida por cada equipo en las cuatro actividades

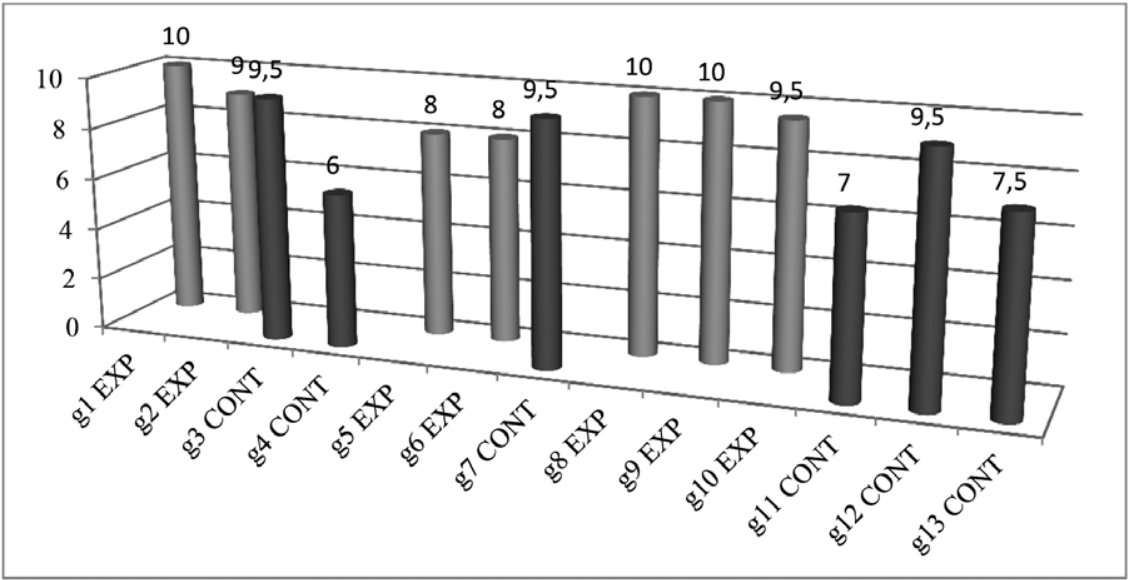

Fuente: elaboración propia.

Gráficos 2 a 5. Puntuaciones obtenidas en las tres evaluaciones realizadas en cada una de las actividades
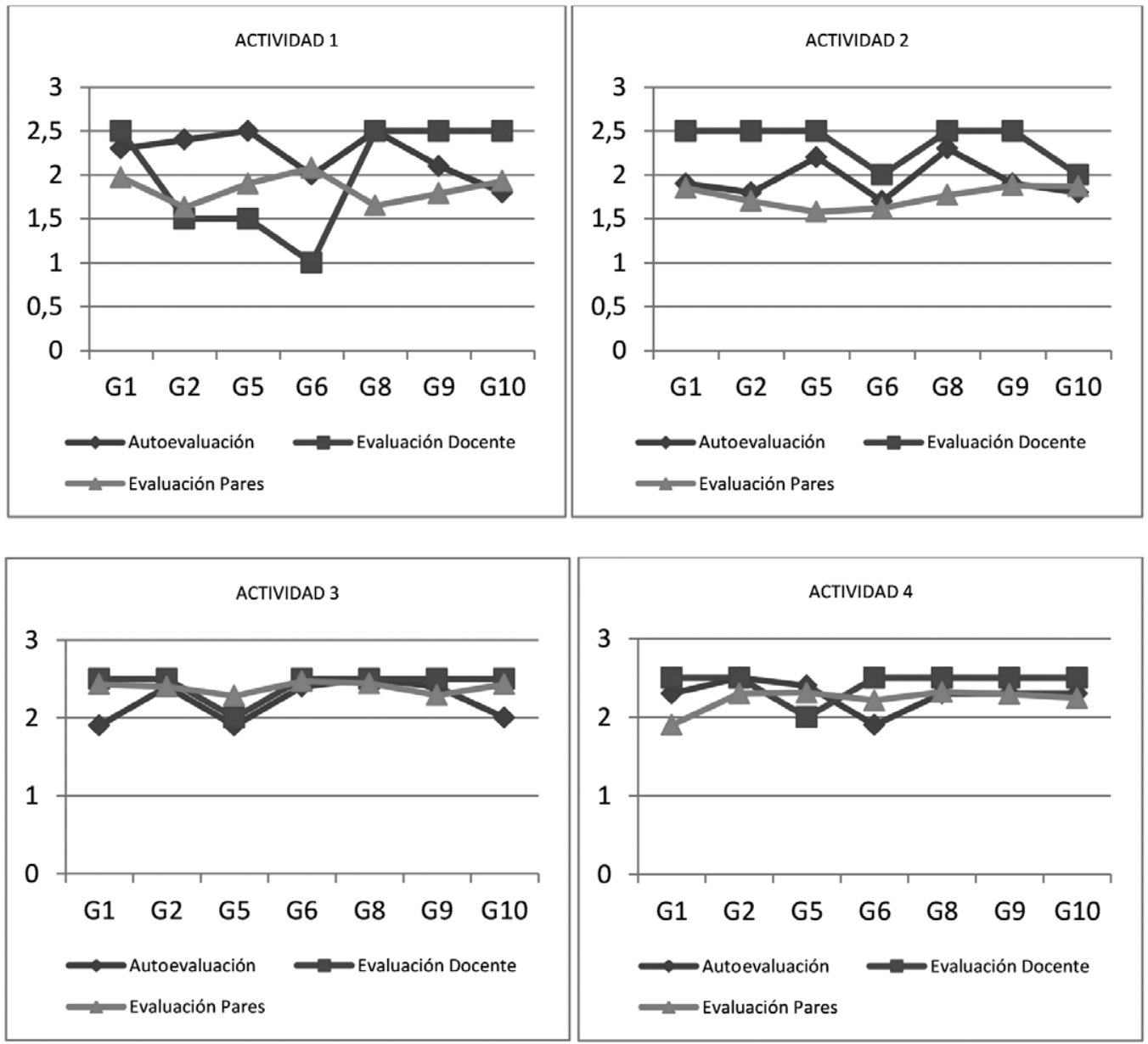

Fuente: elaboración propia. 
Se observa que en las cuatro actividades, los sujetos del grupo experimental van mejorando sus calificaciones con el paso del tiempo. Todos los sujetos parten de una calificación muy semejante, pero en las dos últimas actividades sus notas mejoran acumulándose en los valores más positivos. Se constata también que las puntuaciones suben gradualmente de una actividad a la otra, siendo el incremento mayor en las dos últimas actividades. Esto demuestra una mejora en el rumbo del aprendizaje de contenidos así como en la evaluación de los mismos.

La discrepancia de puntuaciones otorgadas en cada tipo de evaluación (docente, autoevaluación y evaluación de pares) es mayor en las actividades 1 y 2 que en las 3 y 4 . Esto puede ser debido a la novedad de la experiencia para el alumnado. Al mismo tiempo, también podemos observar que a medida que se avanza en la resolución de las actividades hay más coincidencia en las puntuaciones, la diferencia es ínfima, por ejemplo, en la actividad 3, principalmente.

Por lo que respecta a la evaluación de la profesora, las puntuaciones suelen ser más altas proporcionalmente en las cuatro tareas, no sucede lo mismo cuando la evaluación es con los iguales. El alumnado tiende a ser más duro cuando ejerce este proceso de reflexión con otros compañeros, tal vez por un afán desmedido de demostrar su responsabilidad en la tarea. Respecto a la autoevaluación, se percibe el progreso de los grupos en cuanto a su propia percepción; esta suele comenzar con una alta autoevaluación que desciende en la segunda actividad para ir regulándose a medida que se avanza en el curso. Sus percepciones son más realistas. Esto es, se desciende en puntuación en un primer momento para luego ascender y mantenerse. Estos aspectos los vemos reflejados en el gráfico 6, que recoge las puntuaciones medias obtenidas en cada tipo de evaluación.

La evaluación de la docente normalmente es más alta que la realizada por los estudiantes, aunque las diferencias entre los dos agentes se fueron reduciendo a la par que la competencia de evaluación mejoró. Así, a medida que se avanzaba en la realización de las actividades, había un mayor acuerdo en las puntuaciones. Las diferencias fueron disminuyendo a medida que los estudiantes iban logrando una mejor comprensión de su papel, cada vez eran más conscientes de la importancia de los indicadores para su aprendizaje. Estos datos son coherentes con las aportaciones de Biggs (2005), quien sostiene que la autoevaluación y coevaluación no solo mejoran la adquisición de conocimientos, sino que también proporcionan a los estudiantes la oportunidad de mejorar sus procesos metacognitivos.

Gráfico 6. Puntuación media obtenida en cada tipo de evaluación

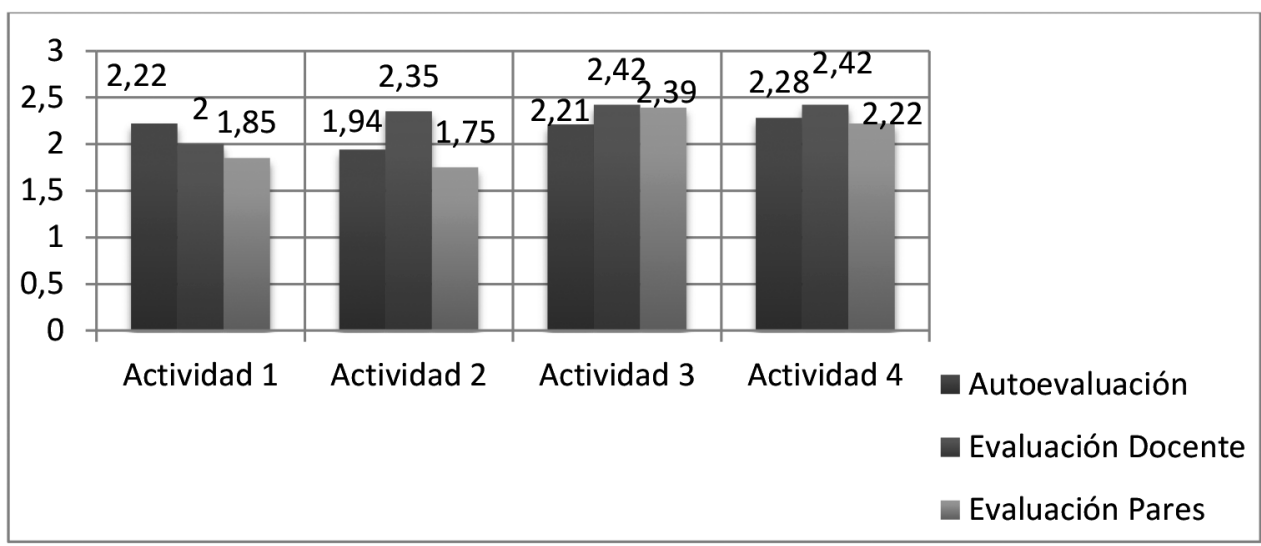

Fuente: elaboración propia. 
Por último, en cuanto a la herramienta empleada, constatamos que las valoraciones de los estudiantes son muy positivas en relación con el uso de rúbricas. Desde su punto de vista, la evaluación mediante rúbricas hace que el proceso evaluativo sea "más justo", "más objetivo" y "más reflexivo" que las formas tradicionales de evaluación. Los resultados obtenidos a partir de un cuestionario de satisfacción (Raposo y Gallego, 2012), con el que se les preguntó por su percepción y valoración de la experiencia, nos aportan las siguientes ideas:

- Sobre el hecho de emplear la rúbrica, a la mayoría (77\%) les ha parecido bien porque: "sirve para mejorar", "sirve para cooperar más y responsabilizarse", "el profesor llega a conocer el modo de trabajo propio de cada uno", "permite dar a conocer lo que se espera", "es otra manera de evaluar el trabajo que hemos hecho", "ayuda a reflexionar sobre el trabajo y ver qué podemos hacer para mejorar", "cada persona puede evaluar su aportación y la del grupo", "son interesantes y ayudan en varios aspectos", "podíamos darnos cuenta de los errores y corregirlos".

- Sobre el valor de la rúbrica en el trabajo de los grupos, se considera que esta ha ayudado para valorar la competencia: trabajar en equipo, porque "al evaluarte a ti mismo y a los compañeros se ve más claro", "si alguien no hace algo queda plasmado", "así se ve realmente lo que es trabajar en equipo", "ayuda a reflexionar y valorar en general el trabajo realizado", "no solo me ayudó la rúbrica, sino que también me ayudó el sentido de equipo", "se incluían ítems que lo explicaban bien, que permitían entender lo que se pretende con esta competencia".

- Sobre algunas dificultades con el uso del instrumento, se ha señalado que "algunos compañeros se muestran indiferentes hacia la rúbrica" y que "no coincidimos en los resultados".

\section{Conclusiones}

El proceso de cambio que vive la educación superior demanda también nuevos procesos de evaluación. Uno de los objetivos fundamentales del estudio fue experimentar una metodología basada en la evaluación a través de la rúbrica, tanto de competencias generales y del trabajo en grupo como específicas de la materia implicada en la experimentación. En este estudio nos aproximamos a los efectos que pueden tener las rúbricas en la evaluación educativa realizada por el docente y el alumnado. Este último, desde dos perspectivas: autoevaluándose o evaluando a sus iguales. Así, se facilita una práctica poco generalizada - la evaluación- (Cebrián, Serrano y Ruiz, 2014), competencia que tendrán que desarrollar los estudiantes en algún momento de su futura vida profesional.

Los resultados han mostrado que las rúbricas de evaluación han permitido clarificar el alcance del proceso formativo de los estudiantes, donde a medida que avanza el curso y los estudiantes se familiarizan con el uso de esta herramienta, los resultados de sus evaluaciones (tanto relativas a los compañeros como a sí mismo) se aproximan a la percepción de la docente cuando inicialmente la distancia era mayor. Esto ha permitido, en palabras de Bartolomé, Martínez-Figueira y Tellado (2014), que los futuros docentes hagan suyos los criterios de evaluación, propiciando una mayor autonomía y autorregulación del proceso de aprendizaje. Además, en esta evaluación que realizan los estudiantes, encontramos diferencias importantes a medida que se utilizan las rúbricas: vemos una sustancial mejoría tanto en el aprendizaje de los contenidos de la materia como en el dominio de las competencias vinculadas al trabajo en grupo y la evaluación de aprendizajes. Sobre este último aspecto observamos dos cuestiones más específicas:

- En la autoevaluación son valoradas características como la facilidad de uso, la simplicidad y la objetividad, así como su potencial para 
mejorar el aprendizaje, a fin de aumentar la autoestima y reforzar actitudes como la responsabilidad.

- En la coevaluación se fomenta el aprendizaje "desde" y "con" los demás. Asimismo, se espera que mediante la participación en la coevaluación, la participación en clase mejore.

Un valor añadido de esta experiencia sobre el uso de las rúbricas en los procesos formativos de futuros docentes es que se coadyuva al desarrollo de dos de las competencias clave para enseñar enunciadas Perrenoud (2004): gestionar la progresión de los aprendizajes y trabajar en equipo.

Del mismo modo, la experiencia desarrollada, junto con los resultados positivos de la investigación, convierten a la rúbrica en un recurso válido para innovar las prácticas pedagógicas evaluadoras orientadas a la mejora de la calidad de la educación. Se torna en una herramienta que, tal como hemos visto, permite la mejora de los aprendizajes, y produce un cambio sostenible en la práctica educativa, una evaluación formativa (Cebrián, 2014) y centrar la enseñanza en el aprendizaje de los estudiantes.

El estudio llevado a cabo ha reflejado que la docente implicada en la investigación ha tenido una actitud positiva hacia el empleo de rúbricas, lo que coincide con la confirmación que Kutlu, Yildirim y Bilcam (2010) realizan sobre esta tendencia en el profesorado. A su vez, se pone de manifiesto una actitud similar por parte de los estudiantes, quienes resaltan algunas de las ventajas que esta herramienta les aporta. Se podría decir que el principal obstáculo para su incorporación al proceso de enseñanza-aprendizaje bien puede venir de la reticencia a asumir un papel activo en su aprender.

Al igual que confirman Gallego y Raposo (2014) y Raposo y Gallego (2012), los estudiantes valoran mayoritariamente como positivo el hecho de utilizar rúbricas. Han constatado beneficios por el empleo de las mismas en sus evaluaciones, conside- rando que estamos ante un recurso válido, eficaz y pertinente para utilizar de manera complementaria otros recursos, por ejemplo, los blogs, el portafolios, etc., favoreciendo la adquisición de competencias (Bartolomé et al., 2014).

En unos casos, las razones que dan para dicha valoración tienen que ver con el procedimiento, ya que indican que es rápido, cómodo, objetivo, sencillo, diferente. Algunas afirmaciones al respecto son: "permite valorar de una forma más sincera", "por la posibilidad de feedback", "es una forma rápida de evaluar a los compañeros y a nosotros mismos". Otras razones destacan los aprendizajes realizados, señalando, por ejemplo: "es muy bueno que empecemos a evaluarnos a nosotros mismos y también a los demás para poder aprender y darnos cuenta de cómo podemos mejorar", "aprendemos a ser objetivos y a tener un criterio", "sirve para mejorar y reflexionar sobre lo que has hecho bien y lo que has hecho mal", "nos ayuda a ser críticos y fijarnos en nuestros errores", "siempre está bien saber la opinión de los demás respecto a tu trabajo", "es interesante evaluar a los compañeros porque vemos la dificultad que tiene". Por último, otro grupo de razones se refieren al propio contenido de la rúbrica, al señalar: "permite evaluar a todos con los mismos criterios", "permite conocer cómo se ve desde fuera y no solo el punto de vista del profesor", "tanto el docente como el alumnado sabe lo que se valora".

Unos pocos (13) valoran como indiferente la experiencia, con argumentos como: "está bien la valoración del grupo, de clase y de la profesora pero no es una cosa indispensable", "creo que no se toman en cuenta". Por último, solo ocho opinan mal sobre su uso, debido al carácter cerrado del instrumento ("no se puede hacer ninguna aclaración"), o al rol desempeñado por los propios estudiantes ("hay preferencias entre los compañeros" o "hay gente que puede mejorar su nota puntuándose alto a sí misma").

Respecto a las limitaciones, dificultades e inconvenientes, más que al instrumento, se refieren a las ca- 
racterísticas de la propia evaluación: "al autoevaluarse se tiende a ver solo lo bueno", "puedes ponerte más nota de la merecida", "hay personas que mienten".

Por último, es importante señalar que la rúbrica ha estado y está en un proceso de revisión continua, teniendo en cuenta que su finalidad es la evaluación de competencias para mejorar los grados de aprendizaje, convirtiéndola, como indica Blanco (2008), en un instrumento transversal útil, abierto, dinámico y flexible, además de un recurso ágil y coherente que, coincidiendo con Etxabe et al. (2011), im pulsa el aprendizaje a través de la autoevaluación.
Este enfoque, tal como señalan Cebrián, Serrano y Cebrián (2014), exige que los alumnos reflexionen y autoevalúen su aprendizaje, lo que requiere una mayor participación de los estudiantes en su propia educación y en la autorregulación del proceso de aprendizaje. Esto supone situarse en un enfoque formativo de la evaluación, en un sistema de evaluación por competencias asociado a diseños curriculares y pedagógicos emergentes y actuales, lejos de, como dirían Cebrián, Serrano-Angulo y Ruiz-Torres (2014), un uso generalizado de las rúbricas como instrumentos para la evaluación de los resultados y las calificaciones.

\section{Referencias}

Ahumada, P. (2005). Hacia una evaluación auténtica del aprendizaje. México: Paidós.

Bartolomé, A., Martínez-Figueira, M. E. y Tellado-González, F. (2014). La evaluación del aprendizaje en red mediante blogs y rúbricas: ¿complementos o suplementos? Revista de Docencia Universitaria, 12 (1), 159-176. Recuperado 10 de enero de 2014 de http://red-u.net/redu/index.php/REDU/article/view/767

Biggs, J. (1996). Assessing Learning Quality reconciling institutional staff and educational demands. Assessment and evaluation in Higher Education, 21 (1), 5-16

Blanco, A. (2008). Las rúbricas: un instrumento útil para la evaluación de competencias. En Prieto, L. (coord.), La enseñanza universitaria centrada en el aprendizaje: estrategias útiles para el profesorado (pp. 171-188). Barcelona: Octaedro-ICE de la Universidad de Barcelona.

Bordas, M. I. y Cabrera, F. A. (2001). Estrategias de evaluación de los aprendizajes centrados en el proceso. Revista Española de Pedagogía, 218, 25-48.

Carneiro, R., Lefrere, P., Steffens, K.y Underwood, J. (2011). Self-regulated learning in technology enhanced learning environments: A european perspective. The Netherlands: Sense Publishers.

Cebrián, D., Serrano, J. y Cebrián, M. (2014). Federated eRubric service to facilitate self-regulated learning in the European University Model. European Educational Research Journal 13 (5), 575-583. Recuperado og de enero de 2014 de http://dx.doi.org/10.2304/eerj.2014.13.5.575.

Cebrián, M. (2008). La evaluación formativa mediante e-rúbricas. INDIVISA - Boletín de Estudios e Investigación. Monografía X, 197-208.

Cebrián, M. (2009). Formative and peer-to-peer evaluation using a rubric tool. In Méndez-Vilas, A., Solano, A., Mesa, J. A. y Mesa, J. Research, Reflections and Innovations in Integrating ICT in Education (pp. 60-64). Badajoz: Formatex. 
ISSN $0123-1294$ | Educ.Educ. Vol. 17. No. 3 | Septiembre-Diciembre de 2014 | pp. 499-513.

Universidad de La Sabana | Facultad de Educación

Cebrián, M. (dir.). (2011). Servicio federado de e-rúbrica para la evaluación de aprendizajes universitarios. Memoria Técnica para proyectos tipo A y B. Referencia EDU2010-15432.

Cebrián, M. (coord.). (2012). E-rúbrica federada para la evaluación de los aprendizajes. En Leite, C. y Zabalza, M. (coords.), Ensino Superior. Inovaçao e qualidade na docencia (pp. 405-486). Porto: Universidade do Porto.

Cebrián, M. (2014). El e-portafolios de evidencias y la evaluación formativas con e-rúbricas. Revista de Educação a Distância - EmRede -, 1 (1),7-20. Recuperado 10 de enero de 2014 de http://goo.gl/viga84

Cebrián, M., Serrano-Angulo, J.. y Ruiz-Torres, M. (2014). Las e-Rúbricas en la evaluación cooperativa del aprendizaje en la Universidad. Revista Comunicar, XXII (43), 153-161. Recuperado 9 de enero de 2014 de http:// dx.doi.org/10.3916/C43-2014-15

Daura, F. T. (2013). El contexto como factor del aprendizaje autorregulado en la educación superior. Educación y Educadores, 16 (1), 109-125. Recuperado 23 de octubre de 2013 de http://educacionyeducadores.unisabana. edu.co/index.php/eye/article/view/2139/3042

Delgado, A. M., Borge, R., García, J., Oliver, R. y Salomón, L. (2006). Competencias y diseño de la evaluación continua y final en el espacio europeo de educación superior. Informe del Programa de Estudios y Análisis del Ministerio de Educación de España. Recuperado 16 de junio de 2013 de http://nevada.ual.es:81/ufid/archivos/ competencias\%2oevaluacion\%2oeees-MEC.pdf

Etxabe, J. M., Aranguren, K. y Losada, D. (2011). Diseño de rúbricas en la formación inicial de maestros/as. Revista de Formación e Innovación Educativa Universitaria, 4 (3), 156-169.

Fallows, S. J. y Chandramohan, B. (2001). Multiple approaches to assessment: Reflections on use of tutor, peer and selfassessment. Teaching in Higher Education, 6 (2), 229-246.

Gallego Arrufat, M. J. y Raposo-Rivas, M. (2014). Compromiso del estudiante y percepción del proceso evaluador basado en rúbricas. Revista de Docencia Universitaria, 12 (1), 197-215.

Hafner, J. C. y Hafner, P. M. (2003). Quantitative analysis of the rubric as an assessment tool: An empirical study of student peer-group rating. International Journal of Science Education, 25 (12), 1509-1528.

Ibarra, M. S., Rodríguez, G. y Gómez, M. A. (2012). La evaluación entre iguales: beneficios y estrategias para su práctica en la universidad. Revista de Educación, 359, 206-231.

Kutlu, O., Yildirim, O., Bilcam, S. (2010). The comparison of the views of teacher with positive and negative attitudes toward rubrics. Procedia Social and Behavioral Sciences, 9, 1566-1573.

Martínez-Figueira, M. E., Tellado, F.y Raposo-Rivas, M. (2013). La rúbrica como instrumento para la autoevaluación: un estudio piloto. Revista de Docencia Universitaria, 11 (2), 373-39o.

Mora, J. G. (2004). La necesidad del cambio educativo para la sociedad del conocimiento. Revista Iberoamericana de Educación, 35, 13-37. Recuperado 16 de junio de 2013 de http://www.rieoei.org/rie35ao1.htm 
Olmos-Migueláñez, S. (2008). Evaluación formativa y sumativa de estudiantes universitarios: aplicación de las tecnologías a la evaluación educativa. Salamanca: Ediciones Universidad de Salamanca, Colección Vítor, 228.

Olmos-Migueláñez, S. y Rodríguez-Conde, M. J. (2010). Diseño del proceso de evaluación de los estudiantes universitarios españoles: ¿responde a una evaluación por competencias en el Espacio Europeo de Educación Superior? Revista Iberoamericana de Educación, 35, 1-13. Recuperado 8 de septiembre de 2013 de http:// www.rieoei.org/deloslectores/3757Olmos.pdf

Perrenoud, Ph. (2004). Diez nuevas competencias para enseñar. Barcelona: Graó.

Raposo, M. y Gallego, M. J. (2012). Evaluación entre pares y autoevaluación basadas en rúbricas. En Leite, C. y Zabalza, M. A. (coords.), Ensino Superior. Inovação e Qualidade na docência. Porto. CIIE - Centro de Investigação e Intervenção Educativas (pp. 444-453). Porto: CIIE.

Raposo-Rivas, M. y Martínez-Figueira, M. E. (2011). La rúbrica en la enseñanza universitaria: un recurso para la tutoría de grupos de estudiantes. Revista Formación Universitaria, 4 (4), 19-28.

Raposo-Rivas, M., Cebrián, M. y Martínez-Figueira, M. E. (2014). Electronic rubrics to assess competences in ICT subjects. European Educational Research Journal, 13 (5), 584-594. Recuperado 10 de enero de 2014 de http://dx.doi.org/10.2304/eerj.2014.13.5.584.

Stevens, D. D. y Levi, A. J. (2005). Introduction to rubrics. An assessment tool to save grading time, convey effective feedback, and promote student learning. Canadá: Stylus Publishing.

Tierney, R. y Simon, M. (2004). What's still wrong with rubrics: Focusing on the consistency of performance criteria across scale levels. Practical Assessment, Research \& Evaluation, 9 (2). Recuperado 13 de octubre de $2013 \mathrm{de}$ http://pareonline.net/getvn.asp? $\mathrm{v}=9 \& \mathrm{n}=2$

Trainor, A. A. y Bal, A. (2014). Development and preliminary analysis of a rubric for culturally responsive research. The Journal of Special Education, 47 (4), 203-216.

Valenzuela Zambrano, B. y Pérez Villalobos, M. V. (2013). Aprendizaje autorregulado a través de la plataforma virtual Moodle. Educación y Educadores, 16 (1), 66-79. Recuperado 10 de diciembre de 2013 de http://educacionyeducadores.unisabana.edu.co/index.php/eye/article/view/2000/3039

Wamba, A. M., Ruiz, C., Climent, N.y Ferreras, M. (2007). Las rúbricas de evaluación de los Practicum como instrumento de reflexión para los estudiantes de Educación Primaria. En Cid, A. et al. (coords.), Buenas prácticas en el Practicum (pp.1251-1261). Santiago de Compostela: Imprenta Universitaria.

Weimer, M. (2002). Learner-centered teaching: Five key changes to practice. San Francisco: Jossey-Bass. 
